1965

\title{
Classical Mechanics and Quantum Mechanics
}

Patrick A. Heelan

Georgetown University, heelanp@georgetown.edu

Follow this and additional works at: https://fordham.bepress.com/phil_research

Part of the Philosophy of Science Commons

\section{Recommended Citation}

Heelan, Patrick A., "Classical Mechanics and Quantum Mechanics" (1965). Research Resources. 22.

https://fordham.bepress.com/phil_research/22

This Article is brought to you for free and open access by the Hermeneutic and Phenomenological Philosophies of Science at DigitalResearch@Fordham. It has been accepted for inclusion in Research Resources by an authorized administrator of DigitalResearch@Fordham.

For more information, please contact considine@fordham.edu. 


\section{CLASSICAL MECHANICS AND QUANTUM MECHANICS}

\section{SECTION I: QUANTUM AND CLASSICAL ANALOGUES}

\section{Classical Analogue of Quantum Mechanics}

The discussion begun in the preceding chapters raises the further question of how quantum mechanics is related to classical mechanics. We have insisted that quantum mechanical variables are differently defined from those of classical physics, not only because of the noncommutation of operators, but also because quantum mechanics includes in an organic way the functions of both a deterministic and statistical classical theory. Quantum and classical physics, and the variables appropriate to each are analogous to one another. We shall now examine the analogues which exist between these two branches of physics, and explain in what way classical mechanics is a limiting case of quantum mechanics.

From the differences in theoretical structure between classical mechanics and quantum mechanics, it is clear that the classical numerical variable cannot be the analogue of the quantum mechanical operator, for the quantum mechanical operator represents an individual measurement. The analogue of the quantum mechanical operator (applied to a particular state function) is a concrete instance of a classical variable. The classical numerical variable, as we have already explained, represents not a concrete instance, but an ideal norm or mean from which concrete values do not systematically diverge. No set of measured values of mass, acceleration and force obeys, with infinite precision, Newton's mechanical laws, for Newton's Laws belong, like the whole of classical physics, to the abstract and ideal, and not to the concrete. It is otherwise with quantum mechanics, for it is precisely individual and concrete instances which are envisaged. These are envisaged, however, as a virtual ensemble, that is, each instance is considered concretely as a sample of one chosen at random from a set 
of similar states. In classical mechanics the virtual ensemble is replaced by a single mean value; in quantum mechanics the actual instances, or, more exactly, the frequency of random instances, of the same type are retained. Quantum mechanics, through the matrix form of its operator and of its state-function (or state vector), deals simultaneously with the spectrum of eigen values and their ideal frequencies of occurrence. The correct classical analogue of quantum mechanics is, consequently, a theory formed by substituting for the numerical variables of classical mechanics commuting operators and for the idealised classical particle a virtual ensemble of concrete individual classical particles. The classical analogue of quantum mechanics is not Newtonian particle mechanics, but a statistical theory of classical particles with a built-in "theory of errors".

Alternatively, the same classical analogue of quantum mechanics would be obtained directly from quantum mechanics by letting $h$ (Planck's constant) tend to zero, retaining at the same time the correspondence rules of quantum mechanics 1 . This leads to the wellknown Liouville's equation for classical mechanics, viz., ${ }^{2}$

$$
\frac{\partial}{\partial t} f(p, q, t)=-\frac{\partial H}{\partial P} \cdot \frac{\partial f}{\partial q}+\frac{\partial H}{\partial q} \cdot \frac{\partial f}{\partial p}
$$

where $f(p, q, t)$ is the probability density of the virtual classical ensemble.

In the early days of the quantum theory both Heisenberg and Bohr spoke much of Korrespondenzdenken without ever defining precisely what this meant. Korrespondenzdenken (or the Correspondence Principle) claimed to regulate the relation between classical and quantum physics. In recent years, however, the nature and even the existence of a Correspondence Principle has been much disputed. For that reason, we shall devote the following section to it.

\section{SECTION II: THE CORRESPONDENCE PRINCIPLE}

\section{Various Uses}

According to Heisenberg, Bohr and other representatives of "orthodox" quantum physics, quantum physics is related to classical physics

1 In this chapter a correspondence rule is taken to be a rule for interpreting the mathematical formalism of a physical theory in operational or observational terms. Other names in use are "epistemic rules", "rules of interpretation", etc. There is no connection between correspondence rules and the Correspondence Principle.

2 E. Wigner, Phys. Rev., xL (1932), P. 42; Cf. also F. Bopp, Werner Heisenberg und die Physik unserer Zeit, p. I 36; L. de Broglie, Non-Linear Wave Mechanics, Pp. I66-I7o. 
through the Correspondence Principle. What is the Correspondence Principle?

The Correspondence Principle is fundamentally the statement that quantum physics should be consistent with classical physics and that the results of quantum physics should pass in some way into classical results in limiting cases ${ }^{1}$. However, there are many different views about what precisely is the essence of the Correspondence Principle. It has even been said that no such principle exists, that it is simply the name given to the clue which helped Bohr and Heisenberg to find an acceptable generalisation of classical physics ${ }^{2}$. Heisenberg himself nowhere formulates the Correspondence Principle in precise language, but he allows himself to be guided now by one form, now by another, all of which reflect different applications of what he means yb Korrespondenzdenken. The following are five of the principal formulations of the Correspondence Principle used or implied by Heisenberg in his work.

(a) The correct form of the quantum mechanical equation is suggested by the classical analogue of the quantum problem. For example, the general prescription for quantising a classical Hamiltonian is to substitute a linear quantum operator for each of the classical variables ${ }^{3}$.

(b) Because of the statistical interpretation of quantum mechanics, the expectation values of dynamical variables, in the time-dependent case, should obey classical laws ${ }^{4}$.

(c) Usually but not always, the formulae of the quantum theory should pass over into the corresponding classical formulae whenever $h$ (Planck's constant) can be neglected 5,

(d) Usually but not always, the formulae of the quantum theory should pass over to the corresponding classical formulae whenever the

1 "The Correspondence Principle... postulates a detailed analogy between the quantum theory and the classical theory appropriate to the mental picture employed. This analogy does not merely serve as a guide to the discovery of formal laws..., it also furnishes the interpretation of the laws", Heisenberg, Physical Principles etc., p. ro5; cf. also P. A. M. Dirac, The Principles of Quantum Mechanics (Oxford: 1958), P. 84; G. Ludwig, Die Grundlagen det Quantenmechanik (Berlin: 1954), chap. I; N. R. Hanson, Concept of the Positron, chaps. IV-viri.

2 For example, P. K. Feyerabend writes: "no proof is yet available to the effect that existing theories contain the classical point mechanics as a special case", "Problems in Microphysics", in Frontiers of Science and Philosophy, p. $25 \mathrm{I}$.

3 Cf., note 1 above; also Heisenberg, Physical Principles etc., p. I05; Dirac, loc. cit, p. 84.

4 Heisenberg, Physical Principles etc., pp. 89, 94-95, 37-38; Dirac, loc. cit., p. I2 I.

5 Heisenberg, loc. cit., P. IOI; Weyl, Philosophy of Math. etc., PP. 185-186; Dirac, loc. cit., p. 87; that the principle is not universal was pointed out by Bohr in Atomic Theory and the Description of Nature, p. 87. 
quantum numbers are large and the spacing between the eigenvalues is negligible, as, for example, when the size of the system is large 1.

(e) In marginal cases between micro- and macrodomains, both the quantum theory and the classical theory should be valid; and to the extent that the theoretical formulae represent the same act (or acts) of measurement or observation the values predicted by both theories should agree ${ }^{2}$.

The variety of formulations of the Correspondence Principle is derived from the variety of uses made of it by Heisenberg and other classic authors of the quantum theory. Let us assume that there is one which is more basic than the others and capable of founding all the others; then it is evident from the non-necessary character of (c) and (d) that these are secondary and derived principles. Principle (a) generally results in (b), but (b) must be regarded as more basic than (a); for in the case of a clash between (a) and (b), it is (a) that would have to yield. However, as (b) applies only to time-dependent cases and the quantum theory is often and even principally concerned with stationary states, we do not think that (b) is sufficiently broad.

Principle (e) states a certain material correspondence of the quantum theory with classical theory when applied to marginal cases. The condition is not primarily one of continuity of form but of continuity of subject matter, and continuity of certain results. As the nature of a marginal case makes it impossible to distinguish sharply in it quantum from classical subject-matter, (e) must be regarded as a necessary consequence of the demand for the consistency of results in physics. Moreover, the requirement that, where theoretical formulae correspond (through correspondence rules) with the same act (or acts) of measurement, the predicted values should agree, implies a certain continuity of form too. The continuity of form is not a mere symbolic analogy (though this may provide a valuable clue) but the practical agreement of results, taking into account possibly different symbolic forms and different correspondence rules. Moreover, if (e) holds, then (b) also holds in the marginal domain, for in this case the quantum wave packet cannot be much greater than the size of the marginal object, and consequently the expectation values of its dynamical variables based upon a sampling of an ensemble of independent cases will be a good estimate of the classical dynamical variable: the classical variable,

1 Heisenberg, loc. cit., PP. 83, I16; that the principle was not universal was pointed out by Bohr, loc. cit., pp. $69-70,85$.

2 Inplied in Heisenberg, loc. cit,, pp. 66, I05, I07; and by Bohr, loc. cit., Pp. x4, I8, 37, 72; and by Dirac, loc. cit., p. 84. Cf. also Heisenberg, Philosophic Problems etc., p. 24. 
as we have repeatedly said, is itself an ideal norm like an expectation value. Also, if (e) holds, then both (c) and (d) hold in those cases where the theoretical quantum formulae represent the same acts of measurement as the corresponding classical formulae. This last condition, moreover, specifies when and under what circumstances (c) and (d) hold, and also when they do not hold. Principle (e) allows us to respect the difference in symbolic form and the difference in correspondence rules, and at the same time to specify clearly how two such different physical theories can correspond in some principle.

We propose then to regard (e) as the most basic and fundamental expression of the Correspondence Principle. From the logical point of view, the domain of correspondence between classical and quantum physics, which we described as that of "marginal subject matter", is defined in terms of activities, events and data presented and described in the World of observations, and hence possessing a reality independently of which of the two theories is chosen to explain them ${ }^{1}$.

\section{SECTION III: COMPLETENESS OF QUANTUM MECHANICS}

\section{Completeness Principle}

Closely connected with the Correspondence Principle is the problem of the completeness of quantum mechanics as an explanation of physical systems. The Completeness Principle in question is one which affirms the sufficiency of a complete set of commuting variables to provide the maximum amount of information about a system. It does not say that we know when a set of such variables is exhaustively complete. The principle concerns rather the exclusion from the members of a complete set of any variable which is conjugate to a member of the set. Physicists sometimes speak also of the observationa? completeness of a physical theory, meaning the ability to construct apparatus to correspond with any given Hermitian operator - for an arbitrary Hermitian operator is thought to define an observable. The observational completeness of the quantum theory is, according to Feyerabend, "not far from being a myth" 2 . However, this is really of little importance since, unlike the supporters of operationalism (and presumably Feyerabend here?), we do not hold that observables are generalisations of experimental procedures, but that they are the

1 N. R. Hanson expresses almost the same idea in Patterns of Discovery, p. 156.

2 Feyerabend, "Problems of Microphysics", Frontiets of Science and Philosophy, p. 25 I. 
explanations why certain experimental procedures are measureinents of a single property and others are not.

The quantum mechanical state function is unambiguously defined by a complete set of commuting variables. This set contains only one of every pair of non-commuting variables; for example, if one chooses to specify the state-function of a system by the position variable, then its momentum does not enter directly into its specification at all 1 . A complete set of commuting variables describes the number of independent dynamical degrees of freedom of a physical system, and hence the number of initial conditions which must be known if the system is to be described with the degree of completeness permitted by the theory. It would at first sight appear that classical mechanics gives a more complete explanation of the physical system than quantum mechanics, since the number of independent dynamical degrees of freedom considered by classical mechanics is larger. Heisenberg and the majority of quantum physicists, however, have always defended the position that the quantum theory is the more general and complete physical theory. This was the subject of the first major debate between quantum physicists of the Copenhagen School and representatives of the classical viewpoint which took place at the Fifth Physical Conference of the Solvay Institute at Brussels in October ,I927 ${ }^{2}$. Bohr and Einstein were the principal participants and the discussion continued fitfully for many years. The debate was to a certain extent inconclusive and in recent years it has been revived with renewed vigour ${ }^{3}$.

The problem under discussion can be restated in the following way: assuming that quantum mechanics is valid universally in the physical domain, does it include everything which an explanation according to classical mechanics would give? Heisenberg, Bohr and the majority of physicists answer: Yes! Einstein and a small group of physicists say: No!

The principal difficulty is to explain why the quantum mechanical

1 Since a quantum mechanical system represents and is represented by a virtual ensemble, the position variable is specified when the wave function is given; this however leads to a probability density for all possible values. The momentum enters indirectly in this specification since, by a Fourier transform, one can pass from the coordinate representation to the momentum representation.

2 Niels Bohr gives a detailed account of this debate in Albert Einstein: Philosopher-Scientist, ed. by P. A. Schilpp, pp. $201-24 I$.

3 For example: A. Landé, From Dualism to Unity in Quantum Physics (Cambridge: 1960); Foundations of the Quantum Theory, $A$ Study in Continuity and Symmetry, (New Haven: I955); D. Bohm, Causality and Chance in Modern Physics, (Princeton: I957); L. de Broglie, Non-Linear Quantum Mechanics (Amsterdam: r960), as well as a vast literature some of which was referred to on p. 96 , note 6 . 
description of a system does not always pass over into its expected classical description when $h$ (Planck's constant) tends to zero. Although the quantum theory is a "rational generalisation of the causal spacetime description of classical physics, this view does not mean, however, that classical electron theory may be regarded simply as the limiting case of a vanishing quantum of action" 1. Einstein, for example, considered the case of a small perfectly elastic sphere of "marginal" size, bouncing back an forth between two parallel and perfectly elastic walls. The quantum mechanical description is a stationary state in which the probability of finding the ball at any position is a constant 2 . Allowing $h$ to tend to zero does not yield the classical description of a ball moving continuously back and forth between the walls.

We answer Einstein's difficulty by pointing out that the difference between classical and quantum mechanics is not merely the finite size of Planck's constant, but also the correspondence rules. If we let $h$ tend to zero in quantum mechanics while retaining the same correspondence rules, we arrive at a classical statistical analogue of the physical system - and not at the classical mechanical description of an individual system. In this respect, Einstein's objection to the claim that classical (deterministic) mechanics is a particular case of quantum mechanics is a valid one. The difference, as Einstein showed, is especially striking when the quantum mechanical system is taken to be in a stationary state. The classical analogue of this situation (when the only change involved is the neglect of $h$ ) is a Gibb's ensemble composed of a system with a ball which at any time could be found anywhere between the reflecting walls with equal probability. The formulae in the quantum mechanical case go over into corresponding formulae in the classical statistical case.

However, if with the neglect of Planck's constant we also change the correspondence rules so as to associate with each (now commuting) operator the average of a series of measurements made within a small interval of time at a definite epoch - this is the measure of the classical value - then the case is no longer time independent, and, by Ehrenfest's theorem, we arrive at the corresponding classical equations of a single particle.

Again, admitting as we do that the quantum mechanical description is a statistical one, we open ourselves to Einstein's principal objection

1 N. Bohr, Atomic Theory etc., p. 87.

2 A. Einstein, "Elementare Ueberlegungen zur Interpretation der Grundlagen der Quantenmechanik", in Scientific Papers Presented to Max Born, pp. 33-40. 
to the comprehensive claims of quantum mechanics, that it does not describe the individual system and so its description is incomplete. Einstein writes: "Quantum mechanics describes a collectivity of systems, not the individual systems. The description by means of the psi-function is in this sense an incomplete description of the single system, not a description of a real state" 1 . The objection is a classical statement of the rationalist viewpoint.

We have already elaborated our answer in the preceding chapters. We may summarise it as follows. A description of a virtual ensemble (through a probability law) is not necessarily an incomplete description of the individual; but it is our way of knowing the individual, with reference first of all to an ideal norm (determining the character of the instances), and then with reference to the relative frequency of deviations from the norm. This is the invariant structure of our scientific knowing. It is found in quantum physics, and also, though in a less obvious way, in classical physics. What Einstein called the "real objective physical description" of single mechanical systems in classical mechanics is non-statistical only to the extent that the description is ideal and abstracts from all actual, existing and individual cases; and it describes any actual individual case only to the extent that the equations of classical mechanics are united to a statistical "theory of errors" and are, to this extent, in the terminology of classical physicists, "non-objective" 2.

Einstein, Podolsky and Rosen had already formulated the same objection, supporting their arguments by a very clever deduction from the very formalism of quantum mechanics itself 3 . They showed that, while experimentally the position and momentum of a particle cannot be measured exactly at the same instant, quantum mechanics allowed - and in fact seemed to require in some cases - that they should have definite values in principle even independently of the act of observation. They concluded that the quantum mechanical description of concrete cases was incomplete. The argument was based upon Einstein's famous definition of "physical reality": "If without in any way disturbing a system, we can predict with certainty the value of a physical quantity, then there exists an element of physical reality corresponding to this physical quantity" 4.

1 Einstein, ibid., p. 40.

2 Max Born has stressed this fact in Science, cxxu (z955), pp. 675-679, and in Werner Heisenberg usw., pp. 103 I 18 .

3 A. Einstein, B. Podolsky, N. Rosen, "Can Quantum Mechanical Description of Physical Reality be Considered Complete?', Phys. Rec., XL (r935), p. 777.

4 Ibid. 
As a definition of the reality of physical properties, it is open to the following criticisms. In the first place, the physical properties of an actually existing system are defined through interactions with other systems within appropriate measuring processes. We have shown that this is an essential part of what is described by physics and cannot be omitted without idealising reality. Hence, Einstein's conclusion that a quantum particle should have, independently of the measuring process, six determinate phase-space coordinates, is not a statement about individual existing systems, but about a certain idealised model (viz., the classical particle model) of them. We might add that while the conscious act of observation is not constitutive of the meaning of reality - and to this extent we can agree with Einstein - still, the physical property is defined in relation to an interaction (viz., a measuring interaction) even though the outcome may not be consciously observed. For us, the conscious act of observation is only a criterion but a necessary one - of the reality for-us of a physical property or system.

In the second place, implied in Einstein's definition is the rationalistic supposition that it belongs to the definition of reality, that a physical property has a definite and precise measure-number to an infinity of decimal places. We hold that this is false for the following reasons: (a) a precise number is always an ideal norm and represents a constructed rule through which we understand many instances in one concept; (b) the measure-number does not belong formally to the physical property but to its observable symbol (which is found formally in the measuring instrument) and naturally only in so far as this is numbered; (c) a numbering is the application of a humanly constructed set of conventional symbols which are conceptual instruments for expressing physical relationships, and such notions as continuity, limit, irrational number, derivative, etc., belong rather to the conceptual instrument as such than to what is represented concretely by the instrument. We concur with J. L. Synge who has remarked: "When properly understood (i.e., as mathematicians understand them) these concepts exist in the human mind and not in nature; it is a meaningless waste of time to debate whether the ratio of two measured lengths is rational or irrational, or whether matter is continuous or discontinuous, because the concepts of irrationality and continuity belong to the world of the intellect, a world of mathematics, and not to the real world in which phenomena occur and are measured by pieces of apparatus" 1 .

1 J. L. Synge, Relativity: The Special Theory (Amsterdam: 1958), p. I64. 


\section{Summary}

Accepting the view that quantum mechanics is validly applicable wherever classical mechanics is applicable, it becomes necessary to explain how they are related to one another. Whenever Planck's constant (h) can be neglected (i.e., when position commutes with momentum) there is a twofold classical analogue of the quantum mechanical system: (a) if the correspondence rules of quantum mechanics are retained, then the classical analogue is a classical statistical theory; and (b) if the correspondence rules are changed to link the operators with a value averaged over a small time interval, then the operator is effectively replaced by a numerical variable and classical particle mechanics is obtained. The ambiguity in the kind of limiting process leading from quantum mechanics to classical mechanics is reflected in the variety of opinions about the nature of the Correspondence Principle. After an analysis of various opinions, we conclude that the most basic formulation of the Correspondence Principle refers to the continuity of formulae when applied to marginal matter, taking into account the differences in correspondence rules between a typical classical theory and quantum mechanics. We then examine the Completeness Principle attacked by Einstein and show that a satisfactory solution of the Einstein-Podolsky-Rosen paradox depends upon the epistemological analysis made in the preceding chapters. 